\title{
MT Literacy—A cognitive view
}

\author{
Sharon O'Brien, Dublin City University, orcid.org/0000-0003-4864-5986 \\ Maureen Ehrensberger-Dow, Zurich University of Applied Sciences, orcid.org/0000-0002- \\ $5538-4000$
}

\begin{abstract}
MT literacy means knowing how MT works, how the technology can be useful in a particular context, and what the implications are of using it for various purposes. As MT usage grows, the necessity for MT literacy also grows. This knowledge forms part of the greater need for digital literacies. In this contribution, we relate MT literacy to the concept of cognitive load in professional translation production and in translator training scenarios. We then move beyond the sphere of translation studies to examine other use-case settings - crisis communication, academic writing and patent publishing - to consider how MT can offer solutions and how MT literacy can impact cognitively in those settings. We discuss how training in MT literacy can empower language professionals and present two proposals for course content designed for MT users in other sectors.
\end{abstract}

\section{Keywords}

MT literacy, cognitive load, use-case scenarios, digital literacies

\section{What is machine translation literacy?}

In their book dealing with the topic of machine translation and global research, Bowker \& Buitrago (2019) present the following definition of machine translation literacy:

In the context of scholarly communication, machine translation literacy refers to a scholar's ability to:

- comprehend the basics of how machine translation systems process texts;

- understand how machine translation systems are or can be used (by oneself or by other scholars) to find, read, and/or produce scholarly publications;

- appreciate the wider implications associated with the use of machine translation;

- evaluate how (machine) translation-friendly a scholarly text is;

- create or modify a scholarly text so that it could be translated more easily by a machine translation system; and

- modify the output of a machine translation system to improve its accuracy and readability.

(Bowker \& Buitrago 2019, 88)

They suggest this as an evolving definition that may not remain definitive, mainly because the notion of machine translation (MT) literacy is novel. The definition lies within their focal point of using MT as a tool for scholarly research and publication, but the definition is surely applicable to many other settings. Basically, MT literacy means knowing how MT works, how it can be useful in a particular context, and what the implications are of using MT for specific communicative needs.

This article explores the concept of MT literacy, positioning it within the broader discussion on digital literacies, while also applying a cognitive lens. We explain why MT literacy is more important than ever to translation production and expand on this by discussing three use cases outside the area of professional translation, exploring certain cognitive dimensions of MT usage in each scenario. In the final part of the paper, we mention two new initiatives that seek to train and empower non-traditional MT users and conclude that such initiatives will need to be expanded as MT usage increases so language professionals should be prepared to provide such training. 


\title{
2. Why MT literacy is important
}

Machine translation has grown in deployment over the past decades, when the paradigm shifted from one of rule-based to data-driven MT and the resulting output was more usable. With a recent shift to neural MT (NMT), the quality has again improved, and languages that were considered too difficult for successful MT are now more viable (e.g., Finnish, Irish). Consequently, the commercial application of MT has grown and this trend is expected to continue (Language Industry Survey 2019). More and more free-to-use MT systems have come online, and there have been (questionable) claims of MT output reaching levels of "human parity" (a very problematic concept; see Läubli et al 2020). As a result, increasing numbers of people are using MT for a wide variety of purposes. There is an increasing supposition that MT is now good enough and can replace the work of professional translators. However, these suppositions are often founded on a lack of MT literacy, i.e., no knowledge of how the technology works, of how best to deploy or evaluate it, or of the risks linked with its use without any human intervention. This is not surprising: if you do not know how the magician performs tricks, they seem amazing. Such consequences might vary from the trivial (the output is unintentionally amusing), to reputation damage (a person or company sounds very foolish because they publish inaccurate MT output), to commercial (people will not buy a product or service because the messaging around it seems shoddy and unprofessional), to very serious (output from MT is wrong, leading to actions that might negatively affect well-being). Just as we need to be educated about social media nowadays, we also need to be educated about the pros, cons and serious pitfalls of MT.

Knowledge of MT is a form of digital literacy. The discussion of 'new literacies' began several decades ago in reaction to increased access to televised content and especially to the internet (Coiro et al 2014). It was an attempt to expand the traditional concept of literacy, which refers to reading and writing, to include more informed consumption of media and information presented in digital form. In the meantime, media and digital literacy have become established terms in a landscape of new and/or multiple literacies. UNESCO defines the latter as follows. ${ }^{1}$

\begin{abstract}
The concept is based on the assumption that individuals 'read' the world and make sense of information by means other than traditional reading and writing. These multiliteracies include linguistic, visual, audio, spatial, and gestural ways of meaning-making. Central to the concept of multiple literacies is the belief that individuals in a modern society need to learn how to construct knowledge from multiple sources and modes of representation.
\end{abstract}

(Seel 2012)

The concept of digital literacy, closely related to MT literacy as defined by Bowker \& Buitrago (2019), has less to do with meaning making and construction of knowledge than with allowing people to make informed choices about the deployment of technology (Langset et al 2018). It emerged in the context of higher education research and has become part of public discourse (Spante et al 2018). Over the past two decades, the understanding of digital literacy has expanded to accommodate the various technological developments that are continuing to drive the digitisation of society. Digital literacy is generally understood as a set of skills and competencies needed to find, interpret, evaluate and handle digital information (see Shopova 2014). According to popular and even to widespread academic opinion, the socalled digital native generation who has grown up with the internet, multimedia and new media is assumed to be digitally literate. However, recent studies have challenged this generalisation (e.g., Ståhl 2017). Being able to use technology does not imply understanding it or being able to transfer one technologyspecific set of skills to another: many people consider themselves digitally literate based on their use of social media. As digital natives become adults and join professional life, it is important to consider potential disparities in their use and understanding of digital technologies.

Langset et al $(2018,25)$ point out that "[d]igital competence [...] compris[ing] digital knowledge, skills, attitude and literacy, has become a key competence in education in most developed countries." The EU has published something referred to as The Digital Competence Framework 2.0, which specifies that all citizens are expected to be competent in the five key areas of: (1) information and data literacy; (2) communication and collaboration; (3) digital content creation; (4) safety; (5) problem solving. ${ }^{2}$ Van Laar et al $(2017,583)$ expand on this slightly in their list of core $21^{\text {st }}$-century digital skills: technical,

\footnotetext{
${ }^{1}$ http://www.ibe.unesco.org/en/glossary-curriculum-terminology/m/multiple-literacies

${ }^{2}$ https://ec.europa.eu/jrc/en/digcomp/digital-competence-framework
} 
information management, communication, collaboration, creativity, critical thinking and problem solving. Each of these skills, including creativity, can be enabled by MT, but MT is rarely seen in such a light.

The recent advances in artificial intelligence, natural language processing and ready access to freely available online translation tools have not gone unnoticed by the general public, resulting in increased pressure on language service providers to lower their prices and on translation training institutions to justify their existence. What the casual user may not realise, though, is that many MT systems produce misleadingly fluent texts (see Martindale \& Carpuat 2018). This can lull those with little training into blindly trusting the translation output, although these systems are far from flawless or always accurate. The sobering reality is that even bespoke MT systems trained with high-quality bilingual corpora (ideally from domain-specific translation memories) still produce output with culturally inappropriate content, errors and ambiguities that could contribute to risk and result in legal consequences (see Wahler 2018, for an analysis of these, including violations of confidentiality incurred by using online systems).

Within translation studies and among the professional translation community, interest in digital literacy with respect to recent advances in NMT has been growing (e.g., Forcada 2017). Translation students are expected to develop the capacity for decision-making on the deployment of language technologies by learning about the capabilities and limitations of the machines and tools they might be working with (see Massey \& Ehrensberger-Dow 2017). The list of competences that are components of MT literacy according to Bowker \& Buitrago (2019) would probably seem familiar to most institutions currently involved in translator training, especially those within the European Master's in Translation network (EMT 2017, 9). MT literacy can inform judgements about the appropriate genres, quality expectations, risks, and limitations that call for intervention by translators (see also Nitzke et al 2019). ${ }^{3}$ Although rarely mentioned in this context, those judgements also necessarily draw on the rich intercultural awareness that translators and other language professionals bring to their work. As intercultural mediators, they have been trained to recognise and deal with cultural differences, appropriateness of register and voice, contextual acceptability, potential ambiguity, terminological inconsistencies as well as conceptual and lexical gaps as they transfer meaning from one language to another (e.g., Federici \& Declercq 2019), issues that MT systems cannot currently resolve.

\section{MT literacy and cognitive load in translation production}

Deploying MT effectively requires that the quality of the output be evaluated and revised as necessary. These cognitive activities demand resources from a capacity that has been assumed to be limited, which is the basic premise of the construct of COGNITIVE LOAD. The construct originated in the field of educational psychology and was proposed by Sweller (1988) to account for how the effort needed to process task instructions seemed to affect how well the task itself (originally mathematical equations) was performed. It seems intuitive now that the clearer instructions are, the faster and more effectively learners can accomplish a given task. Currently, cognitive load theory assumes that there are at least two additive types of load. Most theorists agree on what has been termed "intrinsic" load and "extraneous" load, although some have also included "germane" load (e.g., Paas et al 2003).

The concept of cognitive load was used in relation to post-editing of MT output early on (e.g., O'Brien 2005; 2006). Intrinsic load is related to the nature of the task itself (in this case processing MT output), whereas extraneous load can be attributed to task-external aspects, such as not having enough information about the source of the MT or the output being relatively far away from the source text in the interface being used. Germane load is inherent in well-designed task instructions and facilitates schema construction and automation, which reduce working memory load and thereby allow for effective task completion. With regard to processing MT output, a clear brief with background information as well as a well-structured and well-formulated source text would optimize germane load.

One problem with the construct of cognitive load is that it can only be inferred by assessing the cognitive effort actually expended during a task, which in turn can only be measured via indirect indicators. Reference is often made to Krings (2001) in this type of research, who also proposed technical and temporal effort as important indicators. Within the theoretical framework of situated and embodied cognition, these are encompassed by cognitive effort (e.g., Mellinger 2014, but also see Muñoz 2016), which is associated with the actual responses by the task performer in a particular situation. Although

\footnotetext{
${ }^{3}$ We deliberately refer throughout to 'translators' rather than 'post-editors' when we mean (trained) language professionals because the convergence of CAT tools and MT means that the activities of translation, revision and post-editing are becoming almost inseparable.
} 
cognitive load can theoretically be the same for a particular task, the effort expended during the completion of it depends on the individual's background knowledge, experience and circumstances.

With respect to the task of post-editing MT, we assume that MT literacy can lower cognitive load by making it easier for the individual performing the task to evaluate the output and identify errors or problems. An example from the second author's professional practice can serve as an illustration. An abstract in English was sent by a senior German-speaking academic with a request to proofread it. There were no spelling or punctuation errors, but even a cursory reading showed that a careful revision was needed. Struggling with aspects related to coherence and terminology that seemed more typical of German writing, the reviser suddenly wondered whether this might actually be MT output and, on a hunch, ran the English text through an online NMT system and then found the resulting German text with a simple web search. When asked, the client rather sheepishly admitted to using NMT to produce the English version but, thinking that "certain bits sounded slightly odd", he had asked for the text to be checked. Relieved of the assumptions that a domain expert had produced the English text and that the word choices were wellmotivated, the reviser proceeded to post-edit the abstract to the client's satisfaction and with much less effort than the first attempt at staying true to the style and argumentation had required (a phenomenon also alluded to by Somers 1997: 99). ${ }^{4}$ We can link this experience to two of Bowker \& Buitrago's (2019) components of MT Literacy, i.e., comprehending the basics of how machine translation systems process texts and modifying the output of a machine translation system to improve its accuracy and readability.

A counter example is linked with levels of trust and MT literacy. Those who know about MT systems and how they operate tend to have a healthy level of mistrust in the output. However, this mistrust can also lead to unnecessary cognitive effort. If revisers know that MT was used, they may not necessarily trust the terminology produced by the MT system and may spend considerable time checking and double-checking terms. However, if they are informed that the MT system was trained on a large volume of high-quality, domain-specific data, with additional controls implemented for terminology, then they may be more inclined to trust the terms produced by the MT system and expend their cognitive effort on actual problems.

Going back to our first example, the struggle when the reviser still thought that the English text had been produced by a human and the relief at being free to make her own decisions is reminiscent of the concepts of cognitive friction (Cooper 2004) and flow (Nakamura \& Csikszentmihalyi 2002). Those concepts were drawn on previously to understand the irritation sometimes caused by the tools that translators use (Ehrensberger-Dow \& O'Brien 2015; O'Brien et al 2017) and are referred to here in discussing two studies described below to explore how the concepts might be related to MT literacy and cognitive load in various constellations.

An example of how MT literacy might impact cognitive load can be illustrated by considering the results of a small study with MA translation students that focused on the relative ergonomics of two different CAT tools with integrated MT (Kappus \& Ehrensberger-Dow 2020). Both CAT tools included the possibility of using MT output but differed with respect to where the output was displayed on the screen and the type of MT output. The students were reasonably familiar with the CAT tool in which the MT output appeared as a static suggestion in a separate window in the upper right part of the screen but less so with the tool in which the MT output appeared immediately below the source text segment and adapted constantly as the target text was entered. Nevertheless, they seemed to be able to adjust to the possibilities offered by the adaptive MT solution easily, perhaps because they all had a certain level of MT literacy. They had taken an introductory course in pre-editing, MT and post-editing the previous semester and were in the middle of an advanced course on translation technology, which dealt with the convergence of MT and TM systems.

With respect to the four measures in this study (i.e., total time, number of deletions, number of keystrokes and number of clicks), the students expended less effort when working in the CAT tool that offered adaptive MT output in close proximity to the source text segments. The productivity gains did not seem to come at the expense of the quality, either, since fluency and adequacy ratings of three comparable segments were consistently better for the tool that offered the adaptive MT output. Interestingly, the majority of the participants reported that they perceived the source text translated in the setting with the static MT in a separate window to be harder (and they made little use of those MT suggestions). Since the two source texts were as closely matched as possible, this perception of higher cognitive load might have had less to do with intrinsic load than with the cognitive friction and extraneous load induced by the more complicated user interface. In contrast, the suggestions from the adaptive MT seemed to trigger solutions

\footnotetext{
${ }^{4}$ The reviser explained this to the client and charged for all of the time involved in completing the job, in an attempt to instil some MT literacy.
} 
for the students as they produced their TTs, possibly fostering flow and contributing to higher productivity and quality. This potential of MT to lighten cognitive load and contribute positively to the translation process has not yet been examined to any extent but should certainly be considered in discussions of MT literacy.

Another aspect of the increased use of MT in professional translation and its relationship to MT literacy that deserves to be explored in more detail is the assumption that it compromises creativity. There have been reports in the literature that some professionals are hesitant to incorporate MT into their own practice because it could block their thought processes or creativity (Cadwell et al 2016; 2018). In a study in which professionals translated literary texts both with and without CAT tools and MT, they reported feeling that they could be more creative when not constrained by the segmentation inherent in CAT tools or by overly literal MT output (Moorkens et al 2018). When MT output is convincingly fluent, the temptation to follow the path of least resistance and simply accept it might indeed be greater. The Swiss federal chancellery recently evaluated the usefulness of an NMT system for their language services and concluded that it could be a useful addition to their repertoire of tools. They cautioned, however, that only trained translators would be in a position to know when to trust whether the source text meaning had actually been conveyed in the target text and that even they could be misled by the fluency of the output. ${ }^{5}$ Healthy scepticism is an important component of MT literacy, as discussed in the previous sections, and this caution suggests that MT literacy should be incorporated into translator training and professional development.

Professional translators pay attention to much more than target text fluency and are constantly evaluating possible solutions with respect to considerations such as genre appropriateness, author intention and audience needs. The notion of MT priming, which has been mentioned as a potentially negative influence (e.g., Green et al 2013), could also be perceived as an effective way to activate semantic associations in the target language. Treating the MT output as one possible version of a draft might even encourage translators to generate alternative solutions, which has been identified as a crucial element in the creative process (Bayer-Hohenwarter 2012, 320). However, the presence of the MT output might cause translators to fixate on it with too little regard for the source text (see Elming et al 2014), which could be detrimental to the creative process. In a study with 12 recently-graduated and current MA students, Ciriello (2019) used the criteria proposed by Bayer-Hohenwarter (2012) to evaluate the creativity of translation processes and products translated from scratch or post-edited from NMT output. She found that overall post-editing elicited less creativity than translation from scratch and that there was evidence of fixation on the MT output. Interestingly, though, she also found that there was practically no difference in the creativity scores between translation from scratch and post-edited NMT for the five most creative students. This suggests that people can be trained to use MT output to their creative advantage. Returning to Bowker $\&$ Ciro Buitrago's (evolving) definition, the component "modify the output of a machine translation system to improve its accuracy and readability" $(2019,188)$ could be extended to include "appropriateness and creativity".

\section{MT literacy and cognitive load in other use-case scenarios}

In this section, we focus on some scenarios outside the area of professional translation production and the implications that the use of MT and MT literacy have for cognitive load. Our first example relates to crisis communication, our second to academic writing and the third to the patent process.

\subsection{MT literacy and crisis communication}

Translation in and for emergency scenarios is a topic that had been neglected, but is now receiving growing attention (cf. INTERACT - The International Network in Crisis Translation). ${ }^{6}$ Despite the acknowledgement that "information saves lives" and that timely and accurate information is essential in a crisis, the fact that this information frequently needs to be in more than one language has been overlooked. Translation (including interpreting) is an obvious requirement for all stages of an emergency.

There are already examples of the use of MT for emergency situations, especially for the "response" stage. For example, the response to the Haiti earthquake in 2010 included the rapid building of a Haitian Creole MT system because the international responders could not communicate well with local responders or

\footnotetext{
${ }^{5}$ https://www.newsd.admin.ch/newsd/message/attachments/59735.pdf

${ }^{6} \mathrm{https} / / /$ sites.google.com/view/crisistranslation/home
} 
affected people (Lewis 2010; Lewis et al 2011). In situations where information is being disseminated in large volumes and changing every hour, there is no doubt that people are using online MT systems to translate that information, at least to get a gist of the message.

The use of MT for emergency response could be very beneficial, but also brings with it a number of considerable challenges relating to resources, quality and language coverage. Where resources (professional translators and interpreters in this instance) are low or simply non-existent, it may be tempting for response organisations to just use raw MT output. Without any editing, there is a chance that the message will simply be wrong. While this can lead to amusing examples in the domain of tourism, it can lead to much more serious outcomes in emergencies, including impacts on health and well-being, if not life itself.

Trust in information plays an important role in crisis communication, and this also applies to translated information (Cadwell 2019; Alexander \& Pescaroli 2019). There is evidence from other domains that trust in information is inversely related to cognitive load (i.e. trust decreases in high-load circumstances; see Chen et al 2016 for a review), which can be assumed to be greater in crisis situations. What role does trust play when that information is machine translated and how can we influence trust such that receivers of information know when they can and cannot trust MT output? The relationship between MT and trust has received some attention, since machine-translated outputs are far from flawless and fully accurate even after the quality improvements introduced by the neural paradigm (Toral et al 2018), thus often requiring revision. Research has tended to revolve around approaches to identify machine-translated words, sentences or documents that pass a predetermined quality threshold and are therefore more trustworthy (Soricut \& Echihabi 2010). The focus of this research has not just been on a lack of trust of MT output, but also on "blind trust" in it (Depraetere 2010).

There is a paucity of empirical work on MT and trust, though, especially in relation to emergency communication. Rossetti et al (2020) sought to investigate the relationship between trust and comprehension of MT and non-MT flood warnings. Native speakers of Italian with varied levels of English competence were shown flood warning messages in English and Italian, and their comprehension, trust and potential compliance was measured. This study focussed on levels of trust based on labelling of messages: Although all Italian messages were labelled as 'machine translated', half of them were presented as "post-edited" and half as "raw MT output," even though in reality all had been post-edited to a high standard. This was a small and limited study and, consequently, there were only slight and non-significant differences found in terms of comprehension scores and trust levels across the English and Italian messages. Unsurprisingly, there was a beneficial effect of MT on comprehension and trust among those who had lower competence in English. Labelling text as having been "post-edited" resulted in some improvement in comprehension but, counterintuitively, not in trust levels. The results suggest that there is a need for more research on the impact of MT (and its labelling) on trust and compliance for emergency communication and that the relationships are complex. We presume that trust in MT is closely linked with MT literacy.

\subsection{Academic writing and MT literacy}

In order to disseminate research internationally and to meet increasing requirements for research impact, scholars are expected to publish in international journals, which usually means in the undisputed lingua franca of academia, English (Bennett 2013; 2014; 2015). This leads to considerable disadvantages for those who have not attained a sufficiently high level of academic English. Those disadvantages can be cognitive (increased effort while writing, Breuer 2015), economic (need to pay translators or proofreaders, Lillis \& Curry 2010), and temporal (additional time required for proofreading or translation). It is sometimes the case that proofreaders do not master specialised domain vocabulary, which requires even more time for checking by the author (Willey \& Tanimoto 2015).

Using MT to support academic publication in English could be a solution to some of these problems. Indeed, if non-native authors have some mastery of English, which is more than likely the case for academics, they could write their papers in their L1, machine translate them, and then "self-post-edit" (MT+Self-PE; see Goulet et al 2017; O'Brien et al 2018).

Several questions emerge in this use-case scenario, some of which are directly related to levels of MT literacy and to cognition. One is whether writing a paper in L1 and using MT+Self-PE can be more successful than writing directly in English without any additional aids. "Success" might be measured here by using parameters such as (i) quality of the text produced; (ii) time taken to produce the text; (iii) cognitive effort involved in the different production modalities; (iv) cost of production; (v) publication 
success. For (v), publication success could be measured by considering rejection vs. acceptance of the article as well as comments from editors or reviewers about the clarity and quality of language.

A number of factors come into play here. For instance, what is the English language writing competence of the author to begin with? Presumably, the higher the competence in the production of academic English, the less useful the MT+Self-PE solution will be. Knowing what an MT system can produce and what the author is capable of without using MT is crucial here. Another parameter is the language pair involved. Some language pairs will produce better quality than others. It is reasonable to expect, for example, that with most systems Spanish to English will produce better output than Arabic to English. Furthermore, the distance between the genre of academic writing in L1 and that of English is important. The MT+Self-PE solution places an unusual cognitive demand on the author, i.e., write in L1 but use the academic genre conventions for English rather than those of the L1, because MT systems lack knowledge of genres.

The coverage of the domain by the MT system is an additional parameter. If the domain is particularly specialised, as academic domains tend to be, it may be that the terminology is not covered by the MT system. Related to this point, it is often the case that specialised domain terminology is underdeveloped in some languages. Writing about that domain in their L1 might place significant cognitive demands on authors because the terminology does not exist or they are simply not familiar with it because their domain operates in an English as lingua franca context. One interesting cognitive process that emerges here is code switching while writing, i.e., the terminology is in English while the narrative or descriptive text is in the L1. Some MT systems can deal with this by marking terms for language, effectively telling the system 'this is already in English' or 'do not translate' so that they are dealt with better.

In general, MT literacy would benefit anyone seeking to use MT for academic text production because they would understand why certain mistakes occur and what to look out for while post-editing. There are still quite a few cognitive challenges that need to be considered, as mentioned above, i.e. code-switching, genre switching, error recognition and revision. It may be that MT is a good solution for a 'first draft' that is not just post-edited, but used as a primer to help write a new draft. To date, there has been very little work on the cognitive challenges of using MT for self-publication (although see Goulet et al 2017; O’Brien et al 2018).

\subsection{MT literacy in the patent process}

Nurminen $(2019 ; 2020)$ reports on the use of raw MT output by patent professionals for what she refers to as MT gisting to understand the contents of documents relevant to the patent process and international property rights that are in languages they have little or no competence in. It has proven especially useful in the patent examination process and is explicitly referred to in the European guidelines. ${ }^{7}$ The process ensures that the MT output is clearly marked as such, and the professionals that Nurminen interviewed seemed to have quite a high degree of MT literacy. For example, they appreciated the value of comparing MT output with that of a more recent version of the same system or from a different system (e.g., from other countries' patent offices) in order to facilitate understanding. The patent professionals also seemed to understand the risks associated with using MT and were accustomed to assessing the benefits of MT versus a professional translation, mentioning "the assumed relevance and importance of the other language document and potential consequences if a misunderstanding would occur due to an error in the gist MT" (Nurminen 2019, 35) as important factors.

Nurminen (2020:108) describes the cognitive activity that patent professionals are involved in as "deciphering" MT output in a distributed, coupled system of their knowledge and competences and the MT engine itself. According to her explanation, deciphering basically comprises reading and trying to understand raw MT output, whose quality (and the consequent cognitive load) would depend both on the engine used and the source text. The distributed aspect relates to the patent professionals having access to resources such as domain experts and other MT systems. Although Nurminen does not explicitly mention it in her discussion of distributed cognition in this setting, the patent professionals' MT literacy would be informed by their experience with the MT engine in question and would also inform their decision making with respect to the need to access solutions from a second MT system and their risk assessments about the need for human translation. It was unclear whether the experienced patent professionals in her study were provided with training in the use of MT output or whether they had simply acquired their expertise on the job, although Nurminen (2019: 40) does suggest that further research with less experienced users would be worthwhile. Training scenarios for MT literacy similar to those outlined in the next section could be

\footnotetext{
${ }^{7}$ EPO (2018) Part G, Chap. IV-4.1 https://www.epo.org/law-practice/legal-texts/html/guidelines/e/g_iv_4_1.htm
} 
applied in this context to evaluate its effectiveness in speeding up the acquisition of this type of digital expertise.

\section{Training and empowerment}

An increasing number of translator training programmes are including the topic of MT, and competence in this technology is now seen as a core component of translation competence models. This training empowers professional translators not only to be knowledgeable end users of MT, but also to act as expert advisors to companies and organisations who wish to implement MT in their workflows. This addresses a comment frequently uttered within professional translation communities: "we need to educate the client." MT literacy is, for these graduates, a valuable skill. For other sectors, however, such as those we detailed above and more besides, there is no formal MT literacy training available. We suggest that this kind of training is required to ensure that MT is deployed in a sound manner.

Some limited efforts in training for sectors other than professional translation have begun to emerge, but they are in their infancy. We mention two examples here. The first is the preliminary framework for MT literacy instruction, proposed by Bowker \& Buitrago (2019). They suggest a cluster of modules that could be delivered to researchers who wish to explore the use of MT for scholarly work. Four modules are proposed: (1) Why think about machine translation in the context of scholarly work? (2) Overview of machine translation systems; (3) Translation-friendly writing and editing; and (4) Self-post-editing machine translation output. For each module, they provide a target audience, goals, allotted time and a brief description of the content that could be covered. They suggest that this could be delivered in a halfday workshop format.

The second example is connected with the crisis communication setting discussed in Section 4.1. As with all aspects of crisis response, or even health communication in general (Cadwell et al 2019), volunteerism plays an important role and contributes significantly to official response efforts. Citizens with relevant language competence will offer to translate content for those who have been affected by a crisis and for whom response organisations have not (yet) provided translated information. In this context, translation is, of course, sometimes provided (for free or as a paid activity) by professional translators. However, there may be a shortage of trained professionals for certain language pairs and regions, leading to contributions from volunteers who have little or no training or experience in translation. Furthermore, organisations might resort to using machine translation to fill this gap. This tendency for crisis response, and indeed for other areas, demands that training in MT literacy be provided.

An example of training for machine translation for 'citizen translators' was created by the aforementioned INTERACT project. Although the focus was on training content for crisis settings, the course content is general enough to be applicable to other domains. The course-MTPE101, or Machine Translation and Post-Editing 101 consists of five short online courses on YouTube that are structured as follows. ${ }^{8}$

1. What is MT? This explains the core concepts involved in machine translation.

2. Data and MT Quality. This helps trainees understand the importance of underlying data and how it links with the quality produced by MT systems. This course addresses the issue that users of MT understand that MT makes mistakes, but do not understand why those mistakes occur.

3. Overview of Post-Editing. This is a brief module that explains the core concepts in post-editing.

4. The Task of Post-Editing. This describes basic techniques and individual tasks involved in post-editing machine translation output.

5. Post-Editing Skillset. This course explains the skills required by somebody who might have to edit MT output.

These are just two examples of MT literacy training content that have recently been developed with a specific use case in mind, but which could be extended to broader MT literacy training initiatives. MT literacy training will empower people to make better decisions and potentially lead to more demand for professional translation, or at least fewer bad decisions about deployment of MT.

\footnotetext{
${ }^{8} \mathrm{https}$ ://www.youtube.com/channel/UCRbg0k_1W8KU1xDFqZjDZtg
} 


\section{Conclusion}

In this article we have embraced the theme of MT literacy and explored it from a cognitive perspective, hopefully enriching the initial proposal by Bowker \& Buitrago (2019) on what MT literacy is and why it is important. We positioned the discussion about MT literacy in the broader, ongoing discussion on the necessity of digital literacies. From a translation studies perspective, MT has received a lot of attention in the past decade or so, with research on cognition, ergonomics, productivity, quality, training, impact on the profession and, more recently, creativity. Our aim was to extend this discussion to demonstrate why MT literacy is essential in translator training and how, in fact, it can be advantageous for professional translators above and beyond performing the fundamental task of revision, opening new opportunities for them as consultants or trainers on the appropriate use of MT.

We have suggested that interaction with MT might have an impact on creativity, but that, contrary to common perception, this could be positive as well as negative and that MT literacy training might in fact contribute to increasing creativity for some who are open to that idea. This is very much in line with Earley and Ang's $(2003,9)$ multidimensional concept of cultural intelligence, which they define as "a person's capability for successful adaptation to new cultural settings". The extension of this concept to "intercultural intelligence' captures the reality of translators shifting back and forth between the context of the source text and that of the target text. Conceptualising MT literacy as being at the interface between languagerelated artificial intelligence and intercultural intelligence allows for the integration of apparently opposite poles of the human-machine spectrum. Such a conceptualisation can provide the space to encourage the development of expertise in language technology and at the same time foster the uniquely human dimensions of intercultural mediation, intuition, creativity and ethical judgement.

Additionally, we highlighted some areas outside professional translation where MT is being used and where it is likely to increase. We deliberately selected three pertinent though diverse use cases to demonstrate the broad scope of MT in today's world (crises, academic writing and patent publishing) and show how MT literacy can impact on these operationally and cognitively. There are other use cases that could be included in future discussions, for example, language learning, education from primary through to tertiary level, plagiarism, literary publication, the medical field etc. The need for training in MT literacy has also been identified, with two examples of training proposals and course content provided to indicate useful starting points. Further training developments are required for increasing MT literacy, which, as mentioned earlier in our wider discussion on digital literacies, would allow people to make better informed choices about the deployment of this particular technology. The assumption that the digitally native generation is, in fact, digitally literate has been challenged, as mentioned earlier. Extending this to MT literacy one cannot assume that ease of access to online MT systems automatically results in knowledgeable users of the technology. MT can sometimes be treated as the foe of professional translation. However, by increasing translators' understanding of how it works and when it is appropriate to use it, we anticipate that it will no longer be seen as something to be feared. Evidence suggests that only a small portion of content produced globally is translated (Language Industry Survey 2019) but as MT becomes more successful, translation volumes are increasing globally. Not all of that increasing content is, or should be, machine translated. Increasing MT literacy will assist with the decision as to when it is sensible-from a process, economic and cognitive perspective - to use the technology and when it is not. 


\section{References}

Alexander, David, and Gianluca Pescaroli. 2019. "The Role of Translators and Interpreters in Cascading Crises and Disasters: Towards a framework for confronting the challenges." Disaster Prevention and Management 29 (1): 144-156. https://doi.org/10.1108/DPM-12-2018-0382.

Bayer-Hohenwarter, Gerrit. 2012. Translatorische Kreativität: Definition—Messung—Entwicklung [Translational Creativity: Definition-Measurement—Development]. Tübingen: Narr.

Bennett, Karen. 2013. "English as a Lingua Franca in Academia. Combating epistemicide through translator training." The Interpreter and Translator Trainer 7 (2): 169-193.

Bennett, Karen. 2014. "The Political and Economic Infrastructure of Academic Practice: The "semiperiphery' as a category for social and linguistic analysis." In The Semi-periphery of Academic Writing: Discourses, communities and practices. Edited by K. Bennett, 1-12. London: Palgrave Macmillan. Bennett, Karen. 2015. "Towards an Epistemological Monoculture: Mechanisms of epistemicide in European research publication. In English as an Academic and Research Language (English in Europe Vol. 2). Edited by R. Plo Alastrué, and C. Pérez-Llantada, 9-35. Berlin: De Gruyter Mouton. Bowker, Lynne, and Jairo Buitrago Ciro. 2019. Machine Translation and Global Research: Towards improved machine translation literacy in the scholarly community. Bingley: Emerald Publishing. Breuer, Esther O. 2015. First Language versus Foreign Language. Fluency, errors and revision processes in foreign language academic writing. Frankfurt am Main: Peter Lang.

Cadwell, Patrick. 2019. "Trust, Distrust and Translation in a Disaster." Disaster Prevention and Management 29 (2): 157-174.

Cadwell, Patrick, Claudia Bollig, and Juliane Ried. 2019. "Management and Training of Linguistic Volunteers: A case study of translation at Cochrane German." In Translation in Cascading Crises. Edited by F. M. Federici, and S. O'Brien, 152-173. London: Routledge.

Cadwell, Patrick, Sheila Castilho, Sharon O’Brien, and Linda Mitchell. 2016. "Human Factors in Machine Translation and Post-editing among Institutional Translators." Translation Spaces 5 (2): 222-243.

Cadwell, Patrick, Sharon O'Brien, and Carlos S. C. Teixeira. 2018. "Resistance and Accommodation: Factors for the (non-) adoption of machine translation among professional translators." Perspectives 26 (3): 301-321.

Chen, Fang, Jianlong Zhou, Yang Wang, Kun Yu, Syed Z. Arshad, Ahmad Khawaji, and Dan Conway. 2016. Robust Multimodal Cognitive Load Measurement. Cham: Springer.

Ciriello, Livia. 2019. Post-Editing und Kreativität [Post-editing and Creativity]. Master's Thesis, ZHAW Zurich University of Applied Sciences.

Coiro, Julie, Michele Knobel, Colin Lankshear, and Donald J. Leu. 2014. "Central Issues in New Literacies and New Literacies Research." In The Handbook of Research on New Literacies. Edited by J. Coiro, M. Knobel, C. Lankshear, and D. J. Leu, 1-21. New York; NY: Taylor \& Francis.

Cooper, Alan. 2004. The Inmates are Running the Asylum: Why hi-tech products drive us crazy and how to restore the sanity. Indianapolis: Sams Publishing.

Depraetere, Ilse. 2010. "What Counts as Useful Advice in a University Post-Editing Training Context? Report on a case study." $14^{\text {th }}$ Annual Meeting of the European Association for Machine Translation. Accessed April 19, 2020. http://www.mt-archive.info/10/EAMT-2010-Depraetere-2.pdf.

Earley, P. Christopher, and Soon Ang. 2003. Cultural Intelligence. Individual interactions across cultures. Stanford, CA: Stanford Business Books.

Ehrensberger-Dow, Maureen, and Sharon O'Brien. 2015. "Ergonomics of the Translation Workplace: Potential for cognitive friction." Translation Spaces 4 (1): 98-118.

Elming, Jakob, Laura W. Balling, and Michael Carl. 2014. "Investigating User Behaviour in Post-editing and Translation using the CASMACAT Workbench." In Post-editing of Machine Translation: Processes and applications. Edited by S. O'Brien, L. W. Balling, M. Carl, M. Simard, and L. Specia, 147-169. Newcastle upon Tyne: Cambridge Scholars.

EMT. 2017. European Master's in Translation Competence Framework 2017. Brussels: European Commission.

Federici, Federico M., and Christophe Declercq, eds. 2019. Intercultural Crisis Communication. London: Bloomsbury.

Forcada, Mikel L. 2017. “Making Sense of Neural Machine Translation.” Translation Spaces 6 (2): 291309.

Goulet, Marie-Josée, Michel Simard, Carla Parra Escartín, and Sharon O’Brien. 2017. "La traduction automatique comme outil d'aide à la rédaction scientifique en anglais langue seconde: résultats d'une étude exploratoire sur la qualité linguistique. [Machine Translation as an aid for ESL Academic Writing: 
Results of an exploratory study on linguistic quality]." La revue du GERAS 72: 5-28. DOI

10.4000/asp.5045.

Green, Spence, Jeffrey Heer, and Christopher D. Manning. 2013. "The Efficacy of Human Post-editing for Language Translation." Proceedings of the 2013 ACM SIGCHI Conference on Human Factors in Computing Systems (CHI), 439-448. https://doi.org/10.1145/2470654.2470718.

Kappus, Martin, and Maureen Ehrensberger-Dow. 2020/forthcoming. "The Ergonomics of Translation Tools: Understanding When Less is Actually More." The Interpreter and Translator Trainer.

Krings, Hans P. 2001. Repairing Texts: Empirical Investigations of machine translation post-editing processes. Edited by G. S. Koby. Kent, OH: Kent State University Press.

Langset, Inger Dagrunn, Dan Yngve Jakobsen, and Halvdan Haugsbakken. 2018. "Digital Professional Development: Towards a collaborative learning approach for taking higher education into the digitalized age." Nordic Journal of Digital Literacy 13 (1): 24-39.

Language Industry Survey. 2019. Expectations and Concerns of the European Language Industry. Accessed April 19, 2020. https://euatc.org/wp-content/uploads/2019/11/2019-Language-Industry-SurveyReport.pdf.

Läubli, Samuel, Sheila Castilho, Graham Neubig, Rico Sennrich, Qinlan Shen, and Antonio Toral. 2020.

"A Set of Recommendations for Assessing Human-Machine Parity in Language Translation." Journal of Artificial Intelligence Research 67: 653-672. https://doi.org/10.1613/jair.1.11371

Lewis, William. 2010. "Haitian Creole: How to build and ship an MT engine from scratch in 4 days, 17 hours, \& 30 minutes." $14^{\text {th }}$ Annual Meeting of the European Association for Machine Translation.

Accessed April 19, 2020.

https://pdfs.semanticscholar.org/9083/5bc87814208e303ebb3e8b58eed03aa4063e.pdf?_ga=2.126917512.4 45420217.1587735964-1033436181.1587330375.

Lewis, William, Rob Munro, and Stephan Vogel. 2011. "Crisis MT: Developing a Cookbook for MT in Crisis Situations." Proceedings of the $6^{\text {th }}$ Workshop on Statistical Machine Translation 501-511. Accessed April 19, 2020. https://www.aclweb.org/anthology/W11-21.

Lillis, Theresa, and Mary Jane Curry. 2010. Academic Writing in a Global Context: The politics and practices of publishing in English. London: Routledge.

Martindale, Marianna J., and Marine Carpuat. 2018. "Fluency over Accuracy: A pilot study in measuring user trust in imperfect MT." Proceedings of AMTA 2018 1: 13-25. Accessed April 19, 2020.

http://aclweb.org/anthology/W18-1803.

Massey, Gary, and Maureen Ehrensberger-Dow. 2017. "Machine Learning-Implications for translator education." Lebende Sprache 62 (2): 300-312.

Mellinger, Chris D. 2014. Computer-assisted Translation: An empirical investigation of cognitive effort. Doctoral Dissertation, Kent State University.

Moorkens, Joss, Antonio Toral, Sheila Castilho, and Andy Way. 2018. "Translators' Perceptions of Literary Post-editing Using Statistical and Neural Machine Translation.” Translation Spaces 7 (2): 240262.

Muñoz Martín, Ricardo. 2016. Of Minds and Men-Computers and translators. Poznań Studies in Contemporary Linguistics 52 (2): 351-381. https://doi.org/10.1515/psicl-2016-0013.

Nakamura, Jeanne, and Mihaly Csikszentmihalyi. 2002. "Flow Theory and Research." In The Oxford Handbook of Positive Psychology. Edited by C. R. Snyder, and S. J. Lopez, 89-105. Oxford: Oxford University Press.

Nitzke, Jean, Silvia Hansen-Schirra, and Carmen Canfora. 2019. "Risk Management and Post-editing Competence." The Journal of Specialised Translation 31: 239-259.

Nurminen, Mary. 2019. "Decision-making, Risk, and Gist Machine Translation in the Work of Patent Professionals." Proceedings of the $8^{\text {th }}$ Workshop on Patent and Scientific Literature Translation. Accessed April 19, 2020. https://www.aclweb.org/anthology/W19-7204.pdf.

Nurminen, Mary. 2020/forthcoming. "Raw Machine Translation Use by Patent Professionals. A case of distributed cognition." Translation, Cognition \& Behavior 3 (1): 100-121.

O'Brien, Sharon. 2005. "Methodologies for Measuring the Correlations between Post-Editing Effort and Machine Translatability." Machine Translation 19: 37-58. https:/doi.org/10.1007/s10590-005-2467-1. O’Brien, Sharon. 2006. "Pauses as Indicators of Cognitive Effort in Post-editing Machine Translation Output." Across Languages and Cultures 7 (1): 1-21. https://doi.org/10.1556/Acr.7.2006.1.1.

O’Brien, Sharon, Maureen Ehrensberger-Dow, Marcel Hasler, and Megan Connolly. 2017. "Irritating CAT Tool Features that Matter to Translators." Hermes Journal of Language and Communication in Business 56: 145-162. Accessed April 19, 2020. https://tidsskrift.dk/her/article/view/97229/146028. 
O'Brien, Sharon, Michel Simard, and Marie-Josée Goulet. 2018. "Machine Translation and Self-postediting for Academic Writing Support: Quality explorations." In Translation Quality Assessment: From Principles to Practice. Edited by J. Moorkens, S. Castilho, F. Gaspari, and S. Doherty, 237-262. Cham: Springer.

Paas, Fred, Juhani E. Tuovinen, Huib Tabbers, and Pascal W. M. Van Gerven. 2003. "Cognitive Load Measurement as a Means to Advance Cognitive Load Theory.” Educational Psychologist 38 (1): 63-71. https://doi.org/10.1207/S15326985EP3801 8.

Rossetti, Alessandra, Sharon O'Brien, and Patrick Cadwell. 2020/Forthcoming. "Comprehension and Trust in Crises: Investigating the impact of machine translation and post-editing." Proceedings of EAMT 2020. Seel, Norbet M. ed. 2012. Encyclopedia of the Sciences of Learning. New York: Springer.

Shopova, Tatiana. 2014. "Digital Literacy of Students and Its Improvement at the University." Journal on Efficiency and Responsibility in Education and Science 7 (2): 26-32.

Somers, Harold. 1997. "A Practical Approach to Using Machine Translation Software.” The Translator 3 (2): 193-212. https://doi.org/10.1080/13556509.1997.10798998

Soricut, Radu, and Abdessamad Echihabi. 2010. "TrustRank: Inducing trust in automatic translations via ranking." $48^{\text {th }}$ Annual Meeting of the Association for Computational Linguistics: 612-621. Accessed April 19, 2020. https://www.aclweb.org/anthology/P10-1063.pdf.

Spante, Maria, Sylvana S. Hashemi, Mona Lundin, and Anne Algers. 2018. "Digital Competence and Digital Literacy in Higher Education Research: Systematic review of concept use." Cogent Education 5 (1). https://doi.org/10.1080/2331186X.2018.1519143.

Ståhl, Tore. 2017. "How ICT Savvy are Digital Natives Actually?" Nordic Journal of Digital Literacy 12 (3): $89-108$.

Sweller, John. 1988. "Cognitive Load during Problem Solving: Effects on learning." Cognitive Science 12 (2): 257-285.

Toral, Antonio, Sheila Castilho, Ke Hu, and Andy Way. 2018. "Attaining the Unattainable? Reassessing claims of human parity in neural machine translation." Accessed April 19, 2020. arXiv:1808.10432.

van Laar, Esther, Alexander J. A. M. van Deursen, Johannes A. G. M. van Dijk, and Jos de Haan. 2017.

"The Relation between $21^{\text {st }}$-century Skills and Digital Skills: A systematic literature review." Computers in Human Behavior 72: 577-588.

Wahler, Madison Elizabeth. 2018. "A Word is Worth a Thousand Words: Legal implications of relying on machine translation technology." Stetson Law Review 48: 109-139.

Willey, Ian, and Kimie Tanimoto. 2015. "'We're Drifting into Strange Territory Here': What think-aloud protocols reveal about convenience editing." Journal of Second Language Writing 27: 63-83.

https://doi.org/10.1016/j.jslw.2014.09.010.

\section{Corresponding author address}

Sharon O’Brien

School of Applied Language and Intercultural Studies,

Dublin City University, Glasnevin Campus,

Dublin 9, Ireland

\section{Bio notes}

Sharon O'Brien, $\mathrm{PhD}$, is Professor of Translation Studies in the School of Applied Language and Intercultural Studies at Dublin City University in Ireland. She teaches translation technology, research methodologies, localisation and about translation in crises. Her main research interests are machine translation, cognitive load, translation processes, and the application of translation to crisis communication.

Maureen Ehrensberger-Dow, $\mathrm{PhD}$, is Professor of Translation Studies in the ZHAW Institute of Translation and Interpreting in Switzerland and teaches on both the BA and MA programmes. Her main research interests are cognitive ergonomics, cognitive load, professional translation workplaces, multilingual text production, translation processes, and various types of digital literacy. 